\title{
Optical Sectioning of Live Mammal with Near-Infrared Light Sheet
}

Feifei Wang ${ }^{1, \dagger}$, Hao Wan ${ }^{1, \dagger}$, Jingying Yue ${ }^{1}$, Mingxi Zhang ${ }^{1}$, Zhuoran Ma ${ }^{1}$, Qinchao Sun ${ }^{1}$, Liangqiong $\mathrm{Qu}^{2}$, Huilong $\mathrm{Ma}^{3}$, Yeteng Zhong ${ }^{1}$, Ye Tian ${ }^{1}$, Guosong Hong ${ }^{1}$, Wen Jung $\mathrm{Li}^{4,5}$, Yongye Liang ${ }^{2}$, Lianqing $\mathrm{Liu}^{5}$ and Hongjie Dai ${ }^{1}$,*

${ }^{1}$ Department of Chemistry and Bio-X, Stanford University, Stanford, CA 94305, USA.

${ }^{2}$ Department of Radiology and BRIC, University of North Carolina at Chapel Hill, Chapel Hill, NC 27599, USA.

${ }^{3}$ Department of Materials Science and Engineering, South University of Science and Technology of China, 518055 Shenzhen, China.

${ }^{4}$ Department of Mechanical and Biomedical Engineering, City University of Hong Kong, Kowloon Tong 999077, Hong Kong.

${ }^{5}$ State Key Laboratory of Robotics, Shenyang Institute of Automation, Chinese Academy of Sciences, Shenyang 110016, China.

$\dagger$ These authors contribute equally to this work.

*Correspondence should be addressed to: hdai@stanford.edu

\begin{abstract}
:
Deep-tissue three-dimensional optical imaging of live mammals in vivo with high spatiotemporal resolution in non-invasive manners has been challenging due to light scattering. Here, we developed near-infrared (NIR) light sheet microscopy (LSM) with optical excitation and emission wavelengths up to $\sim 1320 \mathrm{~nm}$ and $\sim 1700 \mathrm{~nm}$ respectively, far into the NIR-II (1000$1700 \mathrm{~nm}$ ) region for 3D optical sectioning through live tissues. Suppressed scattering of both excitation and emission photons allowed one-photon optical sectioning at $\sim 2 \mathrm{~mm}$ depth in highly scattering brain tissues. NIR-II LSM enabled non-invasive in vivo imaging of live mice, revealing never-before-seen dynamic processes such as highly abnormal tumor microcirculation, and 3D molecular imaging of an important immune checkpoint protein, programmed-death ligand 1 (PDL1) receptors at the single cell scale in tumors. In vivo two-color near-infrared light sheet sectioning enabled simultaneous volumetric imaging of tumor vasculatures and PD-L1 proteins in live mammals.
\end{abstract}

Optical imaging of biological systems capable of high spatiotemporal resolution in vivo and ex vivo has revolutionized biology and medicine for visualization and understanding of structures, functions and dynamic processes at the cellular and even molecular scale ${ }^{1,2}$. To circumvent light scattering by tissues, in vivo 3D imaging by nonlinear two-photon fluorescence microscopy (670- 
$1070 \mathrm{~nm}$ excitation) ${ }^{3-5}$ or three-photon microscopy (1300-1700 nm excitation) ${ }^{6-9}$ has reached penetration depths $\sim 0.7-1.5 \mathrm{~mm}$, benefiting from increased scattering mean free path of the nearinfrared (NIR) excitation employed ${ }^{6}$. Light sheet microscopy (LSM) uses orthogonally arranged planar illumination and wide-field detection, capable of high speed 3D optical sectioning, low photo-damage ${ }^{10,11}$ and volumetrically imaging/tracking with subcellular resolution ${ }^{2}$. Currently the excitation and emission of LSM are mostly in the visible except for two photon excitation in the NIR at $\sim 940 \mathrm{~nm}^{12}$ or three photon excitation at $1000 \mathrm{~nm}^{13}$. Light scattering has limited LSM to imaging small transparent animals, organisms (zebrafish larvae, drosophila larvae, Medaka embryo, C. elegans, etc.), mammalian tissue samples after chemical clearing ${ }^{11,14,15}$, and mouse brain at a depth of $\sim 200 \mu \mathrm{m}$ after craniotomy ${ }^{16}$.

In recent years several classes of fluorescence probes have been developed with emission in the NIR-II window (1000-1700 nm) including carbon nanotubes, quantum dots, organic conjugated polymers and molecular dyes, and rare-earth nanoparticles ${ }^{17-30}$. With suppressed photon scattering and diminished autofluorescence in the long-wavelength region, these probes have facilitated one-photon wide-field ${ }^{17-27}$ or confocal ${ }^{28,29}$ fluorescence imaging in the NIR-II window for mouse models of cardiovascular and brain diseases and cancer ${ }^{20,28,30}$. Non-invasive imaging through the skin, skull and body tissues was achieved, with deep penetration depths and high signal-to-background ratio (SBR). Here, we developed NIR-II LSM using organic dyes and $\mathrm{PbS} / \mathrm{CdS}$ core/shell quantum dot (CSQD) probes, extending excitation and emission to the unprecedented $\sim 785-1320 \mathrm{~nm}$ and $\sim$ 1000-1700 $\mathrm{nm}$ regimes respectively. Suppressed light scattering of both excitation and emission allowed up to $10 \mathrm{~mm}^{3}$ volumetric imaging of highly scattering mouse brain ex vivo with a penetration depth of $\sim 2 \mathrm{~mm}$. Importantly, NIR LSM readily afforded in vivo imaging of mouse tumor models non-invasively, enabling real-time observation of unusual tumor microcirculation, and 3D molecular imaging of immune checkpoint proteins at cellular scale in live mammals.

Our home-built LSM employed multiple switchable lasers with Gaussian beams (658 nm, $785 \mathrm{~nm}$ and $1319 \mathrm{~nm}$ ) cylindrically focused into static light sheets for optical sectioning and an InGaAs camera for orthogonally detecting 900-1700 nm fluorescence (Fig. 1a and see setup details in Supplementary Fig. 1). We adjusted the effective numerical apertures of illumination objectives to produce light sheets with balanced waist thickness $(\sim 10-20 \mu \mathrm{m})$ and Rayleigh length $(\sim 0.5-2.0$ $\mathrm{mm}$ ) (see Methods for light sheet shape analysis), suitable for large scale volumetric imaging with single cell resolution. We employed several biocompatible NIR-II probes, an organic nanofluorophore p-FE ${ }^{28}$ (excitation/emission: 650-850 nm/1000-1300 nm, Fig. $1 \mathrm{~b}$ and Supplementary Fig. 2, dynamic light scattering size $\sim 12 \mathrm{~nm}$ ) and PEGylated PbS/CdS CSQD probes ${ }^{29}$ (excitation/emission: ultraviolet to $1500 \mathrm{~nm} / 1500-1700 \mathrm{~nm}$, Fig. 1b and Supplementary Fig. 2, size $\sim 6.9 \mathrm{~nm}$ ). The two probes were sequentially injected intravenously into a mouse through the tail-vein at an interval of $5 \mathrm{~min}$. We sacrificed the mouse at $30 \mathrm{~min}$ post injection while the probes were still circulating in the vasculature, fixed the brain and placed it in glycerol for ex vivo LSM imaging (see Methods for details). Under the same $785 \mathrm{~nm}$ light sheet (LS) excitation, we were able to clearly image the cerebral vasculatures as a function of tissue depth $Z$ in three 
fluorescence emission windows 850-1000 nm (NIR-I, p-FE emission), 1100-1200 nm (NIR-IIa, pFE emission) and 1500-1700 nm (NIR-IIb, CSQD emission) respectively (Fig. 1c and Supplementary Video 1). This allowed side-by-side comparison (Fig. 1c) of fluorescence LSM imaging in three sub-regions of 850-1700 nm under the same $785 \mathrm{~nm}$ LS excitation. Note that refractive index mismatch during scanning was compensated by linearly moving detection objective (Supplementary Figs 4 and 5) and no photobleaching was observed through this work, owing to the highly photo-stable NIR-II probes and minimal photo damage inherent to LSM ${ }^{10,11}$.

With a $785 \mathrm{~nm}$ light sheet, we observed that the brain tissue imaging depth limit increased (Fig. 1c and Supplementary Video 1), background signal decreased (Fig. 1d) and SBR increased (Fig. 1e) at longer detection wavelength from 850-1000 nm to $1100-1200 \mathrm{~nm}$ and $1500-1700 \mathrm{~nm}$ (Supplementary Video 1). The imaging depth limit (defined as tissue depth at which SBR decreased to $\sim 2$ ) increased from $Z_{\mathrm{SBR}=2} \sim 1.0 \mathrm{~mm}$ to $\sim 2.0 \mathrm{~mm}$ and $\sim 2.5 \mathrm{~mm}$ as emission wavelength increased from $\sim 850 \mathrm{~nm}$ to $\sim 1100 \mathrm{~nm}$ and $\sim 1700 \mathrm{~nm}$ (Fig. 1e and Supplementary Video 1). These were direct results of suppressed scattering of emission photons (scattering $\propto \lambda^{-}$ ${ }^{k}$, where $\lambda$ is wavelength and $k$ is in the range of $0.2-4.0$ for biological tissues ${ }^{20}$ ) under the same $785 \mathrm{~nm}$ excitation. Background signal caused by scattering was the lowest in the 1500-1700 nm emission range at all imaging depths ( $Z$ up to $3 \mathrm{~mm}$, Fig. $1 \mathrm{~d}$ and Supplementary Video 1). For emission in the 850-1200 $\mathrm{nm}$ range, background signal increased with tissue imaging depth up to $Z=\sim 1 \mathrm{~mm}$ due to increased scattering by thicker tissue and decreased upon further increases in depth for $Z>1 \mathrm{~mm}$ (Fig. 1d). The latter was attributed to increased light absorption by thicker tissue that attenuated the background signal.

The lateral full width at half maximum (FWHM) values of the smallest cerebral vasculatures imaged in the three emission regions (850-1000 nm, 1100-1200 nm and 1500-1700 nm, Fig. 1f) at their tissue imaging depth limits of $Z_{\mathrm{SBR}=2}=1.0 \mathrm{~mm}, 2.0 \mathrm{~mm}$ and $2.5 \mathrm{~mm}$ were $\sim 7.2 \mu \mathrm{m}, 9.0 \mu \mathrm{m}$ and $8.3 \mu \mathrm{m}$ respectively. Using an imaging objective with high magnification and numerical aperture (NA), < $5.0 \mu \mathrm{m}$ lateral FWHM was achieved by NIR-LSM imaging by detecting 1500$1700 \mathrm{~nm}$ emission under a $785 \mathrm{~nm}$ LS illumination (Supplementary Fig. 6).

As a light sheet propagated in a scatting medium such as an intralipid phantom ${ }^{31,32}$ mimicking the brain tissue, Monte Carlo simulations ${ }^{33}$ and experiments observed light sheet decaying in intensity in the $X-Y$ plane and spreading in $Z$ due to tissue scattering (Fig. 2a,c,d, Supplementary Figs 7-10), which could hinder optical sectioning capability with reduced imaging field of view in $X-Y$ and lower spatial resolution in $Z$. To circumvent this and maximize the benefit of reduced photon scattering at long wavelengths, we constructed a $1320 \mathrm{~nm}$ light sheet to afford the lowest degree of intensity decay and the least LS thickness broadening relative to the $785 \mathrm{~nm}$ and $658 \mathrm{~nm}$ light sheets (Fig. 2a,c,d, Supplementary Figs 8-10). In the fixed brain tissue, $658 \mathrm{~nm}, 785 \mathrm{~nm}$ and $1319 \mathrm{~nm}$ light sheets propagated over a distance of $\sim 1.3 \mathrm{~mm}, \sim 1.7 \mathrm{~mm}$ and $\sim 4.0 \mathrm{~mm}$ respectively (Fig. 2a), within which imaging of cerebral vasculatures by detecting 1500-1700 nm fluorescence of $\mathrm{PbS} / \mathrm{CdS}$ core-shell quantum dots could still resolve small vessels (FWHM $<10 \mu \mathrm{m})$. 
It constituted a breakthrough in one-photon imaging by exploiting long wavelength excitations up to $1300 \mathrm{~nm}$ (Supplementary Fig. 2). Remarkably, the $1319 \mathrm{~nm}$ light sheet could propagate $>6 \mathrm{~mm}$ to allow imaging of large blood vessels in the highly scattering mouse brain over large field of views (Fig. 2a). Suppressed scattering of longer wavelength LS was also gleaned from $X-Y, X-Z$ or $Y-Z$ cross sectional images (Fig. 2e, Supplementary Fig. 11 and Supplementary Videos 2 and 3), with improved SBR (Fig. 2f) and reduced FWHM of feature sizes along the depth $Z$ direction, corresponding to higher vertical resolution and better sectioning capability along $Z$ (Supplementary Fig. 11e,f).

Light sheet microscopy with both excitation and emission in the 1000-1700 nm NIR-II window minimized scattering and maximized the penetration depth and field of view. High resolution 3D NIR-II LSM sectioning (Fig. 3a and Supplementary Video 4, volume $=810 \mu \mathrm{m} \mathrm{x}$ $648 \mu \mathrm{m} \times 3000 \mu \mathrm{m}, 3 \mu \mathrm{m} Z$ increment in depth) afforded sub-10 $\mu \mathrm{m}$ x $10 \mu \mathrm{m} \times 15 \mu \mathrm{m}$ volumetric resolution (FWHM) (Fig. 1f and Supplementary Fig. 11e,f).

Under the $785 \mathrm{~nm}$ LS excitation, the maximum 1500-1700 nm fluorescence signal detected in the cerebral vasculatures of mouse brain cortex layer as a function of depth $Z$ showed two attenuation regions (Fig. 3e). There was an initial exponential attenuation due to reduction in ballistic and nearly ballistic photons (slightly deflected) emerging through the tissue following the Beer-Lambert law $I_{\text {ball }}=I_{0} \mathrm{e}^{-z / l \mathrm{~s}}$ (where $z$ is imaging depth, $I_{0}$ is initial intensity at $z=0 \mathrm{~mm}$ and $l_{\mathrm{s}}$ is scattering mean free path MFP). This was followed by a slower decay region at deeper $Z$ from which multiply scattered photons diffusing through the brain tissue (diffusive region) were dominant ${ }^{34}$. The MFP $l_{\mathrm{s}}$ extracted (Fig. 3e) was $\sim 400 \mu \mathrm{m}, \sim 477 \mu \mathrm{m}$ and $\sim 639 \mu \mathrm{m}$ for 850-1000 $\mathrm{nm}, 1100-1200 \mathrm{~nm}, 1500-1700 \mathrm{~nm}$ windows respectively. The imaging penetration depth limits (see Supplementary Table 1 for detailed scattering coefficients and MFP comparison) were $\sim 2.5 l_{\mathrm{s}}$ (for $850-1000 \mathrm{~nm}$ emission), $\sim 2.6 l_{\mathrm{s}}$ (for $1100-1200 \mathrm{~nm}$ emission) and $\sim 3.3 l_{\mathrm{s}}$ (for $1500-1700 \mathrm{~nm}$ emission).

The capability of NIR-II LSM performing volumetric imaging through scattering tissues at the $1-10 \mathrm{~mm}^{3}$ scale enabled non-invasive in vivo 3D imaging of protruding features on live mice related to disease models (Fig. 4b), facilitating cellular resolution LS sectioning through intact tissues for mammals. We carried out NIR LSM imaging of subcutaneous xenograft tumors on mice ear and right/left flank of back without invasive surgery or installing optical windows ${ }^{35}$. In vivo hemodynamic imaging of a tumor model used for immunotherapy, murine colorectal MC38 tumors on C57BL/6 mice ear was carried out with the NIR-II light sheet fixed at a $Z$ position $300 \mu \mathrm{m}$ below the top (where the fluorescence signal was first detected) of the tumor $(\sim 8 \mathrm{~mm}$ in diameter, Fig. 4a,b). Time-course LS imaging of the p-FE nanofluorophore immediately following intravenous injection into the mouse tail-vein $(785 \mathrm{~nm}$ excitation, $1000 \mathrm{~nm}$ detection at exposure times $\sim 100-800 \mathrm{~ms}$ ) revealed abnormal microcirculation in tumor. Blood flows in tumor vasculatures were found irregular and intermittent (Fig. 4a,c and Supplementary Video 5), with turning-on and shutting-off behavior, oscillatory/fluctuating flowing patterns and even flow direction reversal in the same vasculature (Fig. 4c, marked by arrows, Supplementary Video 5). 
Intermittent blood flows was only inferred from ex vivo histological methods previously ${ }^{36}$, suggesting unstable pressure within a tumor due to uncontrolled tumor angiogenesis. Further, blood supply time (BST) analysis revealed that the p-FE nanofluorophore gradually leaked out from some vasculatures (in the red-colored region in Fig. 4d) but not from others (in the blackcolored region in Fig. 4d).

To further exploit NIR-II LSM, we performed in vivo two-color molecular imaging and vasculature imaging of PD-L1 expressing MC38 tumor ${ }^{37}$ using a renal excretable organic dye ${ }^{38,39}$ (TT dye: excitation $\sim 785 \mathrm{~nm} / \mathrm{emission} \sim 1000-1200 \mathrm{~nm}$ ) conjugated to anti-PD-L1 antibodies and unconjugated PEGylated PbS/CdS CSQD (excitation 1319 nm/emission 1500-1700 nm) intravenously injected into tumor bearing mice. PD-L1 is an important immune checkpoint protein expressed by tumors as a powerful way of T cell immunity evasion. PD-L1 blocking by antibody immunotherapy is a novel approach to treat various cancers. In vivo PD-L1 imaging is important to fundamental understanding of cancer immunity, and to immunotherapy prognosis since treatment efficacy correlated with PD-L1 levels in the tumor ${ }^{40,41} .24 \mathrm{~h}$ post injection of anti-PDL1-TT dye, we first performed wide-field imaging and observed much brighter anti-PD-L1-TT dye signals in MC38 tumors ${ }^{37}$ (Fig. 4e) than post injection of the unconjugated free TT dye (Supplementary Fig. 12b). Since wide-field imaging only provided 2D projected signals and lacked spatial resolution, we switched to 3D in vivo NIR LSM to profile PD-L1 receptors at various depths of the tumor (Fig. 4f) inside the live mouse, a task only done with tumor biopsies previously 42-44. We observed discrete anti-PD-L1-TT dye labelled features inside tumors with sub-6- $\mu \mathrm{m}$ FWHM in the lateral $X-Y$ plane and sub-15- $\mu$ m FWHM in $Z$ using a 50X detection objective (Supplementary Fig. 13b,d), suggesting cellular scale molecular imaging of PD-L1 in vivo. Meanwhile, we imaged irregular tumor blood vessels engulfing PD-L1 expressing cells upon intravenously injecting PEGylated PbS/CdS CSQD (excitation 1319 nm/emission 1500-1700 nm) circulating in the vasculatures with sub-5 $\mu \mathrm{m} \times 5 \mu \mathrm{m} \times 10 \mu \mathrm{m}$ volumetric resolution (FWHM) (Supplementary Fig. 13c,e). The results suggested tumor extravasation of anti-PD-L1-TT dye (injected $24 \mathrm{~h}$ prior to CSQD injection) and specific targeting PD-L1 expressing cells in the tumor of live mouse.

This work developed 3D near-infrared light sheet microscopy for in vivo and ex vivo deep tissue volumetric imaging through highly scattering biological tissues. Light sheet microscopy with both excitation and emission in the NIR-II window avoided shadows or stripes along the illumination direction by suppressing tissue scattering and adsorption effects encountered by visible LSM ${ }^{45}$. Non-invasive NIR-II LSM enabled in vivo observation in wide-field detection mode with suppressed background, which facilitates dynamic processes tracking and molecular imaging at cellular resolution over millimeter scale simultaneously and could provide a complementary method to two-photon microscopy with lower cost and under less invasive conditions. NIR LSM imaging can be further advanced by developing brighter fluorophores with added colors and applying new configurations of LSM ${ }^{46}$. Recent developments such as noncoherent structured illumination ${ }^{47}$ and optical lattices illumination ${ }^{2}$ could be introduced to 
improve the resolution and contrast of NIR-II LSM. Real time molecular imaging of multiple targets by rapid sectioning 3D tissues of live mammals should become possible.

\section{Methods}

\section{NIR-II fluorescence probes}

This work used an organic nanofluorophore p-FE dye, PEGylated core-shell quantum dots $\mathrm{PbS} / \mathrm{CdS}$ CSQD (see Supplementary Fig. 2), and an organic renal excretable TT dye. p-FE is comprised of organic dyes trapped in amphiphilic polymeric micelles $\sim 12 \mathrm{~nm}$ in size measured by dynamic light scattering ${ }^{28}$. The PEGylated PbS/CdS CSQD was developed recently exhibiting a wide range of excitation wavelength spanning from UV to $\sim 1300 \mathrm{~nm}$, high brightness, biocompatibility and liver excretion ${ }^{29}$. The TT dye ${ }^{38,39}$ exhibits similar spectroscopic properties as the p-FE dye with an excitation $\sim 785 \mathrm{~nm}$ and emission $\sim 1000-1200 \mathrm{~nm}$. The TT dye contains an azide group that can be used for conjugation to PD-L1 antibodies by click chemistry for molecular imaging ${ }^{38,39}$. Purified anti-mouse CD274 (B7-H1, PD-L1) antibody was purchased from GoInVivo (Biolegend, Cat\#124328). Conjugation was done through copper-free click chemistry using linker DBCO-PEG4-NHS purchased from Click Chemistry Tools ${ }^{48}$.

\section{NIR-II light sheet microscope setup}

As show in Supplementary Fig. 1, a laser (Gaussian beam) with wavelength of $658 \mathrm{~nm}, 785$ $\mathrm{nm}$ or $1319 \mathrm{~nm}$ with maximum excitation power of $1.7 \mathrm{~mW}, 11.9 \mathrm{~mW}$ and $8.2 \mathrm{~mW}$ respectively was directed into a spatial filter consisting two achromatic lenses (L3 and L4) and a 50- $\mu$ m pinhole $(\mathrm{PH})$. This spatial filter was introduced to improve the circularity and quality of the illumination beam and to generate uniform light sheet across the field of view. For in vivo imaging, the actual excitation intensity illuminated on mouse was $\sim 65 \mathrm{~mW} \mathrm{~cm}^{-2}, \sim 174 \mathrm{~mW} \mathrm{~cm}^{-2}$ and $\sim 89 \mathrm{~mW} \mathrm{~cm}^{-2}$ for $658 \mathrm{~nm}, 785 \mathrm{~nm}$ and $1319 \mathrm{~nm}$ laser, which is below the safety limit for laser exposure (658 $\left.\mathrm{nm}: 200 \mathrm{~mW} \mathrm{~cm}{ }^{-2} ; 785 \mathrm{~nm}: 300 \mathrm{~mW} \mathrm{~cm}{ }^{-2}, 1319 \mathrm{~nm}: 1000 \mathrm{~mW} \mathrm{~cm}^{-2}\right)^{49}$. The excitations could be selected by removable mirrors (M2-M4). Excitation power was measured by a laser power meter (3A-SH, NOVA II, OPHIR). Before the laser entering a cylindrical lens (CL), a vertically arranged adjustable mechanical slit parallel to the CL was used to adjust the span range of light sheet along $Y$-axis direction (Supplementary Fig. 1). A pair of achromatic lenses (L1, L2) and another slit (S1) was used for adjusting the effective numerical aperture of the objective (for adjusting light sheet shape, see below) before the light was focused on the back focal plane of illumination objective (O1) to form the light sheet illumination.

The light sheet was positioned to pass through a tissue sample or a tumor protrusion on a mouse, fluorescence imaging was done by focusing on and normal to the light sheet plane through a detection objective (O2) and a 200-mm tube lens, using a liquid-nitrogen-cooled InGaAs camera (2D-OMA V, Princeton Instruments) after filtered by selected emission filters. The focal lengths of L1, L2, L3, L4 were $60 \mathrm{~mm}, 100 \mathrm{~mm}, 30 \mathrm{~mm}$ and $60 \mathrm{~mm}$, respectively. All the optical components were made by Thorlabs. For illumination objective, we used a $5 \mathrm{X}$ objective $(\mathrm{NA}=$ 0.15, Nikon LU Plan) or a 10X objective (NA $=0.25$, Bausch \& Lomb Optical Co.). For imaging, we used a $4 \mathrm{X}$ objective (NA $=0.1$, Bausch \& Lomb Optical Co.), a 10X objective (NA $=0.25$, 
Bausch \& Lomb Optical Co.) or a 50X objective (NA = 0.6, Nikon CF Plan) (see Supplementary Table 2 for combinations of objectives for various experiments).

\section{Light sheet shape, resolution and field of view considerations}

Two orthogonal slits were mounted in the illumination arm to adjust the light sheet shape by changing the effective numerical aperture NA and the span range along $Y$-axis direction (Supplementary Fig. 1). To observe the light sheet propagation in glycerol, water, intralipid or brain, the cylindrical lens was rotated by $90^{\circ}$ and $\mathrm{S} 1$ was adjusted to control the light sheet spanning along vertical direction and S2 was used to control the actual numerical aperture (NA) of illumination. This allowed us to form light sheets with adjustable waist thickness and Raleigh length to balance imaging resolution and field of view (FOV). When 10X illumination objective and 50X detection objective were used, the effective NA of illumination was adjusted to be $\sim 0.17$. For $5 \mathrm{X}$ illumination objective and $4 \mathrm{X}$ or $10 \mathrm{X}$ detection objective, the effective NA of illumination was adjusted to be $\sim 0.039$ or $\sim 0.051$.

The effective NA was estimated using NA $=n \sin \alpha=n \sin (\arctan (D / 2 f))$, where $n$ is the refractive index, $\alpha$ is the half of aperture angle, $D$ is the illumination width of light sheet (adjusted by slit S1 for LSM imaging) as it exiting the illumination objective, and $f$ is the focal length of illumination objective. At a given width of slit $\mathrm{S} 1, D$ was measured experimentally by putting a scattering paper close to the aperture of the illumination objective. $D$ was adjusted by $\mathrm{S} 2$ when the cylindrical lens was rotated by $90^{\circ}$ for imaging the side view of the light sheet (Supplementary Fig. 7). The measured light sheet waist and Rayleigh length were consistent with those from theoretical estimations based on the effective NA values (see Supplementary Fig. 7).

The diffraction limited resolution along $Z$ for $10 \mathrm{X}, 0.25$-NA detection objectives was 14.8 $\mu \mathrm{m}(850-1000 \mathrm{~nm}), 18.4 \mu \mathrm{m}(1100-1200 \mathrm{~nm})$ and $25.6 \mu \mathrm{m}(1500-1700 \mathrm{~nm})$ estimated by $n \lambda / \mathrm{NA}^{2}$ 50 . The value $(25.6 \mu \mathrm{m})$ in $1500-1700 \mathrm{~nm}$ window was larger than the measured LS thickness (Supplementary Fig. 7). Therefore, the resolution along $Z$ was dominant by LS thickness under this condition ${ }^{10}$, which is down to $\sim 10 \mu \mathrm{m}$ (Supplementary Fig. 7). Using the 0.5-NA 50X detection objective, the diffraction limited resolution along $Z$ was $2.6 \mu \mathrm{m}(850-1000 \mathrm{~nm}), 3.2 \mu \mathrm{m}$ $(1100-1200 \mathrm{~nm}), 4.4 \mu \mathrm{m}(1500-1700 \mathrm{~nm})$. These analysis suggested that the $Z$ resolution of our current NIR-II LSM is down to $\sim 10 \mu \mathrm{m}$, suitable for resolution along $Z$ at the single cell level. $X$ $Y$ diffraction limited resolution is higher, $\sim 2.3 \mu \mathrm{m}(850-1000 \mathrm{~nm}), 2.8 \mu \mathrm{m}(1100-1200 \mathrm{~nm})$ and $3.9 \mu \mathrm{m}(1500-1700 \mathrm{~nm})$ for the 10X, 0.25-NA detection objective and 0.9 $\mu \mathrm{m}(850-1000 \mathrm{~nm}), 1.2$ $\mu \mathrm{m}(1100-1200 \mathrm{~nm})$ and $1.6 \mu \mathrm{m}(1500-1700 \mathrm{~nm})$ for the 50X, 0.6-NA detection objective (estimated by Rayleigh criteria, $0.61 \lambda / \mathrm{NA}$ ).

The LS waist and Rayleigh length are contradicting factors, optimizing one means degrading performance in the other. The selecting actual NA for each experiment were tradeoffs of these two parameters to obtain uniform LS that are as thin as possible across a large enough FOV. The actual NA and corresponding waist and confocal length for each data set were summarized in Supplementary Table 2 . 


\section{Light sheet microscopy 3D volumetric imaging/scanning}

For 3D NIR-II LSM imaging, as the imaging depth changed, an obvious misalignment of light sheet and working plane of the imaging objective appeared due to refractive index mismatch of the air and tissue. This was compensated by a linear movement of detection objective (Supplementary Figs 4 and 5) concurrent with sample $Z$ position change. The sample scanning in $Z$ and detection objective $Z$ compensation movement was realized by a $3 \mathrm{D}$ translation stage (KMTS50E, Thorlabs) and a single-axis translation stage (MTS50-Z8, Thorlabs), respectively (see Supplementary Fig. 4). Different detection windows were selected by using corresponding longpass and short-pass filters. Synchronous control of 3D translation stage movement and image record was realized using LabView software through a data acquisition card (NI USB-6008, National Instruments). Maximum intensity projections (Fig. 3b,c) and 3D rendering was performed using ImageJ. Multi-color fluorescence images were also merged in ImageJ ${ }^{51}$.

\section{Mouse handling and tumor xenograft}

Mouse handling was approved by Stanford University's administrative panel on Laboratory Animal Care. All experiments were performed according to the National Institutes of Health Guide for the Care and Use of Laboratory Animals. C57BL/6 female mice were purchased from Charles River. Bedding, nesting material, food and water were provided. 6 -week-old C57BL/6 mice were shaved using hair removal lotion and inoculated with $\sim 1$ million MC38 cancer cells on the right flank of back near the hindlimb or on the ear for tumor growth. The sample sizes of mice were selected based on previously reported studies. Mice were randomly selected from cages for all experiments. All relevant data are available from authors. During in vivo imaging, all mice were anaesthetized by a rodent anesthesia machine with $21 \mathrm{~min}^{-1} \mathrm{O}_{2}$ gas mixed with $3 \%$ isoflurane.

\section{Ex vivo NIR light sheet microscopy of mouse brains}

For ex vivo LSM of mouse brains in various NIR-I and NIR-II regions (data in Fig. 1c, Fig. 2e, Supplementary Figs 4, 5, 6 and 11), C57BL/6 mice were firstly injected with $200 \mu \mathrm{L}$ of p-FE with O.D. $=4$ at $808 \mathrm{~nm}$, followed by injection of $200 \mu \mathrm{L}$ PEGylated PbS/CdS CSQD (O.D. $=4$ at $808 \mathrm{~nm}$ ) at 5 min post injection of p-FE. The mouse was sacrificed at 30 min post injection under anesthesia, and brain tissues were taken out and fixed with 10\% neutral buffered formalin at room temperature. After washing in PBS twice, the fixed mouse brain was preserved in glycerol at $4{ }^{\circ} \mathrm{C}$ for further imaging.

For NIR-II imaging mouse brains (data in Fig. 2a and Fig. 3a), C57BL/6 mice were injected with $200 \mu \mathrm{L}$ PEGylated PbS/CdS CSQD (O.D. $=4$ at $808 \mathrm{~nm}$ ) and sacrificed at $30 \mathrm{~min}$ post injection. The brain tissues were taken out and fixed with $10 \%$ neutral buffered formalin at room temperature. After washed in PBS twice, the fixed mouse brain was preserved in glycerol at $4{ }^{\circ} \mathrm{C}$ for ex vivo imaging.

For 5X illumination objective and 4X (for data in Fig. 2a) or 10X (for data in Fig. 1c, Fig. 2e, Supplementary Figs 4, 5 and 11) detection objective, the actual NA of illumination was adjusted to be $\sim 0.039$ or $\sim 0.051$. When $10 \mathrm{X}$ illumination objective and $50 \mathrm{X}$ detection objective were used, the actual NA of illumination was adjusted to be $\sim 0.17$ (Supplementary Fig. 6). The corresponding waists for different wavelengths were shown in Supplementary Fig. 7. Other detailed experimental 
conditions, such as $Z$ scanning increment, exposure time, excitation and emission wavelengths, were summarized in Supplementary Table 2.

\section{In vivo wide-field NIR-II fluorescence imaging}

All NIR-II wide-field fluorescence images (data in Fig. 4e and Supplementary Fig. 12) were recorded using a 2D liquid-nitrogen cooled InGaAs camera (Princeton Instruments, 2D OMA-V, USA). An $808 \mathrm{~nm}$ fiber-coupled diode laser (RPMC Lasers, USA) was used as the excitation source and a filter set ( 850 and 1,000 nm short-pass filter) was applied to filter the excitation light. The actual excitation intensity after passing filters was $\sim 70 \mathrm{~mW} \mathrm{~cm}^{-2}$. The fluorescence signal was collected by two achromatic lenses to the InGaAs camera with different magnifications after filtered by corresponding low-pass and long-pass filters. Two-channel fluorescence images were merged in ImageJ.

\section{In vivo NIR light sheet microscopy of tumors}

C57BL/6 mouse bearing a xenograft MC38 tumor on the ear or right/left flank of the back near the hindlimb was injected with anti-PD-L1-TT dye or free TT dye. The mouse was used for in vivo light sheet microscopy imaging immediately and at 24 hour post injection by placing the mouse on a 3D translation stage (KMTS50E, Thorlabs) after anesthesia. The initial LS illumination position $Z$ below the top of the surface of the protruding tumor was controlled by the 3D motorized translation stage. For monitoring dynamic blood flow at a fixed illumination plane through the tumor (data in Fig. 4a), the camera began recording with a preset exposure time immediately after p-FE (200 $\mu$ L, O.D. $=4$ at $808 \mathrm{~nm})$ or PEGylated PbS/CdS CSQD $(200 \mu L, O . D .=4$ at $808 \mathrm{~nm})$ was injected into the tail vein. When the recorded fluorescence images did not show further changes and reached a steady state, 3D light sheet microscopy was performed to volumetrically imaging the vasculatures or mapping the distribution of anti-PD-L1-TT in the tumor (for Fig. 4f). For dynamic observation of blood flow (Fig. 4a, Supplementary Video 5), a 4X imaging objective lens was used. The illumination was generated by a $5 \mathrm{X}$ illumination objective with actual NA of 0.039 (Supplementary Fig. 7). The PD-L1 receptors were profiled using a 10X (Fig. 4f) and a 50X (Supplementary Fig. 13b-e) detection objective. The $Z$ scanning increment, exposure time and excitation and emission wavelengths were summarized in Supplementary Table 2.

\section{Study of light sheet propagation in different media}

We experimentally compared light sheet propagation in glycerol solutions using light sheets with different NA and excitation wavelengths. These experiments were performed in glycerol containing PEGylated $\mathrm{PbS} / \mathrm{CdS}$ CSQD uniformly dispersed in glycerol. The emission was collected in 1500 - 1700-nm window excited by 658-nm, 785-nm and 1319-nm LS illuminations. In order to directly observe light transmission in glycerol, we rotated the cylindrical lens by $90^{\circ}$ and used mechanical slits to control the actual NA and the spanning range along $Y$ (Supplementary Fig. 1). By so doing, the illumination plane was rotated by $90^{\circ}$ and the light sheet shape can be imaged along the $Y$ direction for its $X-Z$ plane for side view (Supplementary Fig. 7a). In a transparent medium (Supplementary Fig. 7a), our experimentally measured waist and double Rayleigh range of light sheet were consistent with theoretical estimations (Supplementary Fig. $7 b, c)$. 
To study the propagation of the light sheet with wavelength of $658 \mathrm{~nm}, 785 \mathrm{~nm}$ and $1319 \mathrm{~nm}$ in a scattering medium, we performed experimental imaging of LS propagation in intralipid solutions of different concentrations (Supplementary Fig. 8a). Light scattering was apparent as the intralipid concentration increased from $0.00 \%$ to $5.00 \%$ when $658-\mathrm{nm}$ or $785-\mathrm{nm}$ excitation was used. Impressively, 1319-nm LS excitation retained its shape over the longest distance. We further simulated the light sheet propagation in the intralipid phantom by Monte Carlo method based on the method developed by Wang et al. ${ }^{33}$ using the scattering coefficient $\mu_{\text {s }}$ and the anisotropy $g$ estimated by ${ }^{31,32}$ :

$$
\begin{gathered}
\mu_{\mathrm{s}}(\lambda)=0.016 \lambda^{-2.4} \\
\mu_{\mathrm{s}}^{\prime}=10.094 \times \text { conc. }+0.433 \\
\mathrm{~g}(\lambda)=1.1-0.58 \lambda
\end{gathered}
$$

Where $\lambda$ is the wavelength, $\mu_{\mathrm{s}}{ }^{\prime}=\mu_{\mathrm{s}}(1-g)$ is the reduced scattering coefficient and conc. is the concentration of intralipid. These parameters were also summarized in Supplementary Table 1.

The illumination waist measured in water at NA $=0.039$ was used as initial FWHM of incident light in Monte Carlo simulations. The simulated results were consistent with the experimental observations (Supplementary Figs 8 and 9). Generally, the length over which the light sheet transmits by less than $\sqrt{2}$ times the initial waist $\left(w_{0}\right)$ is regarded as the distance useful for imaging ${ }^{52}$. Under this definition, the critical length was larger than $1000 \mu \mathrm{m}$ for the $1319-\mathrm{nm}$ excitation in $1.25 \%, 2.50 \%$ and $5.00 \%$ intralipid solutions, much larger than that of $658-\mathrm{nm}$ and $785-\mathrm{nm}$ cases. Since the scattering coefficient of intralipid can be conveniently adjusted by controlling its concentration, it is a widely used phantom to study photon-material interactions. To study the light sheet propagation in an uniform scattering medium with similar scattering characteristics as mouse brain, we performed simulations using the scattering coefficient of the $2.5 \%$ intralipid and anisotropy factor of the brain measured by Shi et al ${ }^{53}$. We compared the simulation results with our experimental observations in mouse brain in Supplementary Fig. 10. The simulated light propagation in brain was consistent with experimental results of 658-nm, 785$\mathrm{nm}$ and 1,319-nm excitation (Supplementary Fig. 10f-h). The critical distances for uniform illumination were $\sim 210 \mu \mathrm{m}, \sim 320 \mu \mathrm{m}$ and $\sim 1000 \mu \mathrm{m}$ for excitations using $658 \mathrm{~nm}, 785 \mathrm{~nm}$ and $1319 \mathrm{~nm}$ light sheet in mouse brain, respectively (Supplementary Fig. 10f-h).

The LS excitation intensity along incident direction is another important parameter for imaging in scattering tissue, as it affects the transmission distance of excitation in the tissue and determines the illumination field. As the intralipid concentration increased, the intensity along propagation direction attenuated faster but the 1319-nm excitation decayed the slowest compared to the 658-nm and 785-nm excitations (Supplementary Fig. 9j-1). Intensity attenuation was influenced by scattering, absorption and anisotropy of tissue. As the brain had larger anisotropy than intralipid, light sheet transmitted longer in the brain (Supplementary Fig. 10a,b,e). In order to study the illumination field of 658-nm, 785-nm and 1319-nm LS in mouse brain, we performed LSM imaging of a brain tissue at a depth of $Z \sim 200 \mu \mathrm{m}$ along the LS incident direction $X$ for up 
to $1 \mathrm{~cm}$ (data in Fig. 2a,b). Though photons in the LS could propagate for as far as $\sim 6000 \mu \mathrm{m}$ to excite fluorescence in large-diameter vasculatures, only in the initial limited distance that small blood vessels (FWHM $<10 \mu \mathrm{m}$ ) could be observed (Fig. 2a). These limited distances were $\sim 1380$ $\mu \mathrm{m}, \sim 1676 \mu \mathrm{m}$ and $\sim 3900 \mu \mathrm{m}$ for $658-\mathrm{nm}, 785-\mathrm{nm}$ and 1319-nm excitation respectively as longer excitation attenuated slower in mouse brain.

For high-quality optical sectioning in LSM, both uniform light sheet waist and available illumination field need to be ensured across the field of view.

\section{References:}

1. Jain, R. K. Antiangiogenesis strategies revisited: From starving tumors to alleviating hypoxia. Cancer Cell 26, 605-622 (2014).

2. Liu, T.-L., Upadhyayula, S., Milkie, D. E. et al. Observing the cell in its native state: Imaging subcellular dynamics in multicellular organisms. Science 360, eaaq1392 (2018).

3. Helmchen, F. \& Denk, W. Deep tissue two-photon microscopy. Nat. Methods 2, 932-940 (2005).

4. Lapadula, G., Bourdolle, A., Allouche, F. et al. Near-IR two photon microscopy imaging of silica nanoparticles functionalized with isolated sensitized Yb(III) centers. Chem. Mater. 26, 1062-1073 (2014).

5. Alifu, N., Yan, L. L., Zhang, H. Q. et al. Organic dye doped nanoparticles with NIR emission and biocompatibility for ultra-deep in vivo two-photon microscopy under $1040 \mathrm{~nm}$ femtosecond excitation. Dyes Pigments 143, 76-85 (2017).

6. Horton, N. G., Wang, K., Kobat, D. et al. In vivo three-photon microscopy of subcortical structures within an intact mouse brain. Nat. Photonics 7, 205-209 (2013).

7. Ouzounov, D. G., Wang, T. Y., Wang, M. R. et al. In vivo three-photon imaging of activity of GCaMP6-labeled neurons deep in intact mouse brain. Nat. Methods 14, 388-390 (2017).

8. Rowlands, C. J., Park, D., Bruns, O. T. et al. Wide-field three-photon excitation in biological samples. Light-Sci. Appl. 6, e16255 (2017).

9. Liu, J., Wu, R., Li, N. et al. Deep, high contrast microscopic cell imaging using three-photon luminescence of $\beta$-(NaYF4:Er3+/NaYF4) nanoprobe excited by 1480-nm CW laser of only 1.5-mW. Biomed. Opt. Express 6, 1857-1866 (2015).

10. Huisken, J., Swoger, J., Del Bene, F., Wittbrodt, J. \& Stelzer, E. H. K. Optical sectioning deep inside live embryos by selective plane illumination microscopy. Science 305, 1007-1009 (2004). 
11. Dodt, H. U., Leischner, U., Schierloh, A. et al. Ultramicroscopy: three-dimensional visualization of neuronal networks in the whole mouse brain. Nat. Methods 4, 331-336 (2007).

12. Truong, T. V., Supatto, W., Koos, D. S., Choi, J. M. \& Fraser, S. E. Deep and fast live imaging with two-photon scanned light-sheet microscopy. Nat. Methods 8, 757-U102 (2011).

13. Escobet-Montalbán, A., Liu, P., Nylk, J. et al. Three-photon light-sheet fluorescence microscopy. bioRxiv (2018).

14. Chung, K., Wallace, J., Kim, S.-Y. et al. Structural and molecular interrogation of intact biological systems. Nature 497, 332-337 (2013).

15. Tomer, R., Ye, L., Hsueh, B. \& Deisseroth, K. Advanced CLARITY for rapid and highresolution imaging of intact tissues. Nat. Protoc. 9, 1682-1697 (2014).

16. Bouchard, M. B., Voleti, V., Mendes, C. S. et al. Swept confocally-aligned planar excitation (SCAPE) microscopy for high-speed volumetric imaging of behaving organisms. Nat. Photonics 9, 113 (2015).

17. Welsher, K., Liu, Z., Sherlock, S. P. et al. A route to brightly fluorescent carbon nanotubes for near-infrared imaging in mice. Nat. Nanotechnol. 4, 773-780 (2009).

18. Welsher, K., Sherlock, S. P. \& Dai, H. J. Deep-tissue anatomical imaging of mice using carbon nanotube fluorophores in the second near-infrared window. P. Natl. Acad. Sci. USA 108, 89438948 (2011).

19. Hong, G., Lee, J. C., Robinson, J. T. et al. Multifunctional in vivo vascular imaging using nearinfrared II fluorescence. Nat. Med. 18, 1841-1846 (2012).

20. Hong, G. S., Diao, S., Chang, J. L. et al. Through-skull fluorescence imaging of the brain in a new near-infrared window. Nat. Photonics 8, 723-730 (2014).

21. Hong, G. S., Zou, Y. P., Antaris, A. L. et al. Ultrafast fluorescence imaging in vivo with conjugated polymer fluorophores in the second near-infrared window. Nat. Commun. 5, 4206 (2014).

22. Diao, S., Blackburn, J. L., Hong, G. S. et al. Fluorescence imaging in vivo at wavelengths beyond 1500 nm. Angew. Chem. Int. Edit. 54, 14758-14762 (2015).

23. Diao, S., Hong, G. S., Antaris, A. L. et al. Biological imaging without autofluorescence in the second near-infrared region. Nano Res. 8, 3027-3034 (2015).

24. Antaris, A. L., Chen, H., Cheng, K. et al. A small-molecule dye for NIR-II imaging. Nat. Mater. 15, 235-242 (2016).

25. Zhong, Y. T., Ma, Z. R., Zhu, S. J. et al. Boosting the down-shifting luminescence of rare-earth nanocrystals for biological imaging beyond $1500 \mathrm{~nm}$. Nat. Commun. 8, 737 (2017). 
26. Won, N., Jeong, S., Kim, K. et al. Imaging depths of near-infrared quantum dots in first and second optical windows. Mol. Imaging. 11, 338-352 (2012).

27. Naczynski, D. J., Tan, M. C., Zevon, M. et al. Rare-earth-doped biological composites as in vivo shortwave infrared reporters. Nat. Commun. 4, 2199 (2013).

28. Wan, H., Yue, J., Zhu, S. et al. A bright organic NIR-II nanofluorophore for three-dimensional imaging into biological tissues. Nat. Commun. 9, 1171 (2018).

29. Zhang, M., Yue, J., Cui, R. et al. A Bright Fluorescent Probe Emitting at $~ 1600 \mathrm{~nm}$ for in Vivo fluorescence imaging. P. Natl. Acad. Sci. USA (2018).

30. Bruns, O. T., Bischof, T. S., Harris, D. K. et al. Next-generation in vivo optical imaging with short-wave infrared quantum dots. Nat. Biomed. Eng. 1, 0056 (2017).

31. van Staveren, H. J., Moes, C. J. M., van Marie, J., Prahl, S. A. \& van Gemert, M. J. C. Light scattering in lntralipid-10\% in the wavelength range of 400-1100 nm. Appl. Opt. 30, 45074514 (1991).

32. Johns, M., Giller, C. A., German, D. C. \& Liu, H. L. Determination of reduced scattering coefficient of biological tissue from a needle-like probe. Opt. Express 13, 4828-4842 (2005).

33. Wang, L., Jacques, S. L. \& Zheng, L. MCML-Monte Carlo modeling of light transport in multi-layered tissues. Comput. Meth. Prog. Bio. 47, 131-146 (1995).

34. Shi, L. \& Alfano, R. R. Deep imaging in tissue and biomedical materials: using linear and nonlinear optical methods. (CRC Press, 2017).

35. Maeda, A., Leung, M. K. K., Conroy, L. et al. In vivo optical imaging of tumor and microvascular response to ionizing radiation. Plos One 7, e42133 (2012).

36. Nagy, J. A., Chang, S. H., Dvorak, A. M. \& Dvorak, H. F. Why are tumour blood vessels abnormal and why is it important to know? Brit. J. Cancer 100, 865-869 (2009).

37. Tang, H. D., Wang, Y., Chlewicki, L. K. et al. Facilitating T Cell Infiltration in Tumor Microenvironment Overcomes Resistance to PD-L1 Blockade. Cancer Cell 29, 285-296 (2016).

38. Zhang, X. D., Wang, H. S., Antaris, A. L. et al. Traumatic Brain Injury Imaging in the Second Near-Infrared Window with a Molecular Fluorophore. Adv. Mater. 28, 6872-+ (2016).

39. Weizhi, W., Zhuoran, M., Shoujun, Z. et al. Molecular Cancer Imaging in the Second NearInfrared Window Using a Renal-Excreted NIR-II Fluorophore-Peptide Probe. Adv. Mater. 0, 1800106 (2018).

40. Balar, A. V. \& Weber, J. S. PD-1 and PD-L1 antibodies in cancer: current status and future directions. Cancer Immunol. Immun. 66, 551-564 (2017). 
41. Song, M., Chen, D., Lu, B. et al. PTEN Loss Increases PD-L1 Protein Expression and Affects the Correlation between PD-L1 Expression and Clinical Parameters in Colorectal Cancer. Plos One 8, e65821 (2013).

42. Kleinovink, J. W., Marijt, K. A., Schoonderwoerd, M. J. A. et al. PD-L1 expression on malignant cells is no prerequisite for checkpoint therapy. Oncoimmunology 6 (2017).

43. Mahoney, K. M., Sun, H., Liao, X. et al. PD-L1 Antibodies to Its Cytoplasmic Domain Most Clearly Delineate Cell Membranes in Immunohistochemical Staining of Tumor Cells. Cancer Immunol. Res. 3, 1308-1315 (2015).

44. Schats, K. A., Van Vre, E. A., De Schepper, S. et al. Validated programmed cell death ligand 1 immunohistochemistry assays (E1L3N and SP142) reveal similar immune cell staining patterns in melanoma when using the same sensitive detection system. Histopathology 70, 253263 (2017).

45. Itoh, R., Landry, J. R., Hamann, S. S. \& Solgaard, O. Light sheet fluorescence microscopy using high-speed structured and pivoting illumination. Opt. Lett. 41, 5015-5018 (2016).

46. Tomer, R., Lovett-Barron, M., Kauvar, I. et al. SPED light sheet microscopy: Fast mapping of biological system structure and function. Cell 163, 1796-1806 (2015).

47. Keller, P. J., Schmidt, A. D., Santella, A. et al. Fast, high-contrast imaging of animal development with scanned light sheet-based structured-illumination microscopy. Nat. Methods 7, 637-U655 (2010).

48. Zhu, S. J., Yang, Q. L., Antaris, A. L. et al. Molecular imaging of biological systems with a clickable dye in the broad 800-to 1,700-nm near-infrared window. P. Natl. Acad. Sci. USA 114, 962-967 (2017).

49. Matthes, R., Cain, C. P., Courant, D. et al. Revision of guidelines on limits of exposure to laser radiation of wavelengths between $400 \mathrm{~nm}$ and $1.4 \mathrm{mu}$ m. Health Phys 79, 431-440 (2000).

50. Hell, S. W. Far-field optical nanoscopy. Science 316, 1153-1158 (2007).

51. Schindelin, J., Arganda-Carreras, I., Frise, E. et al. Fiji: an open-source platform for biologicalimage analysis. Nat. Methods 9, 676-682 (2012).

52. Power, R. M. \& Huisken, J. A guide to light-sheet fluorescence microscopy for multiscale imaging. Nat. Methods 14, 360-373 (2017).

53. Shi, L. Y., Sordillo, L. A., Rodriguez-Contreras, A. \& Alfano, R. Transmission in near-infrared optical windows for deep brain imaging. J. Biophotonics 9, 38-43 (2016).

\section{Acknowledgments}

This study was supported by the National Institutes of Health NIH DP1-NS-105737. 


\section{Author contributions}

H.D., F.W., H.W. and J.Y. conceived and designed the experiments. H.D. and F.W. designed the optical system. F.W. set up the optical system. F.W., H.W., J.Y., H.M. and Z.M. performed the experiments. M.Z. developed the PbS/CdS CSQD dyes. Q.S. did the Monte Carlo simulations. F.W., H.W., J.Y., H.M., Z.M., Q.S., L.Q., Y.Z., Y.T., G.H., W.J.L., Y.L., L.L. and H.D. analyzed the data. F.W., W.H. and H.D. wrote the manuscript. All authors contributed to the general discussion and revision of the manuscript.

\section{Competing interests}

Authors declare no competing interests.

\section{Materials \& Correspondence}

Correspondence and requests for materials should be addressed to H.D. (hdai@stanford.edu $)$. 
a

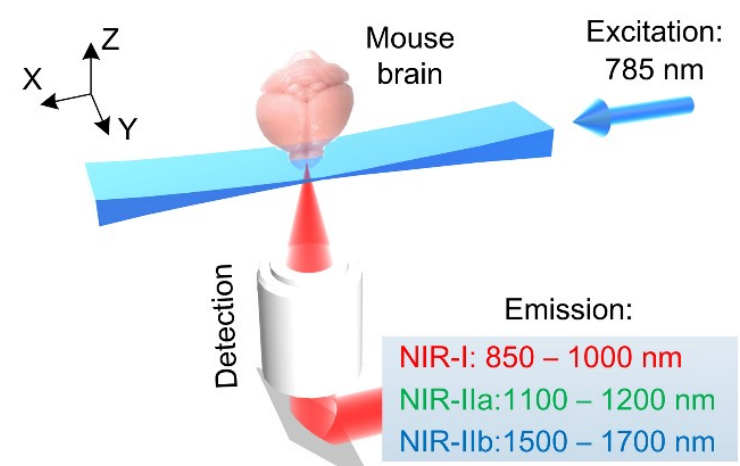

b

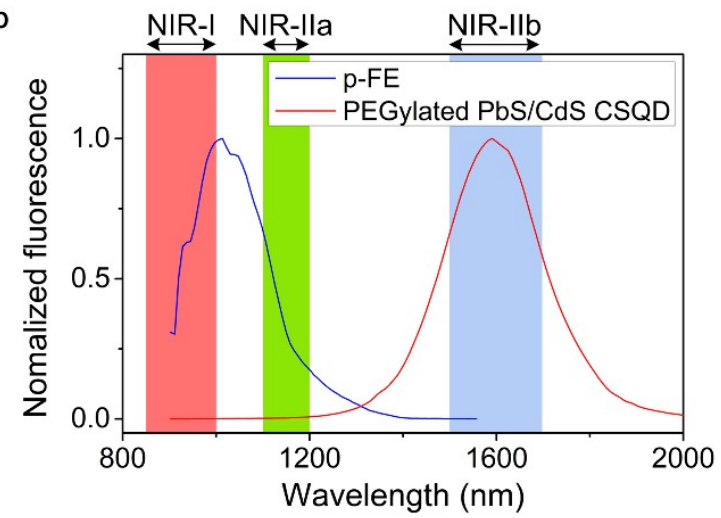

c
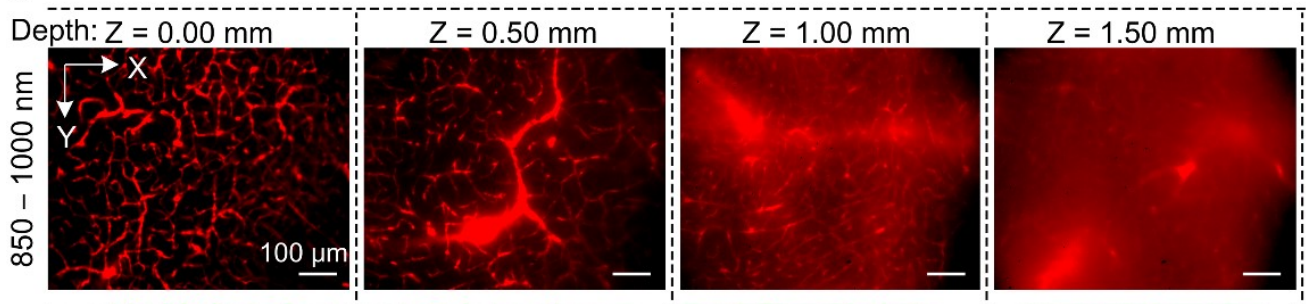

$\mathrm{Z}=2.00 \mathrm{~mm}$
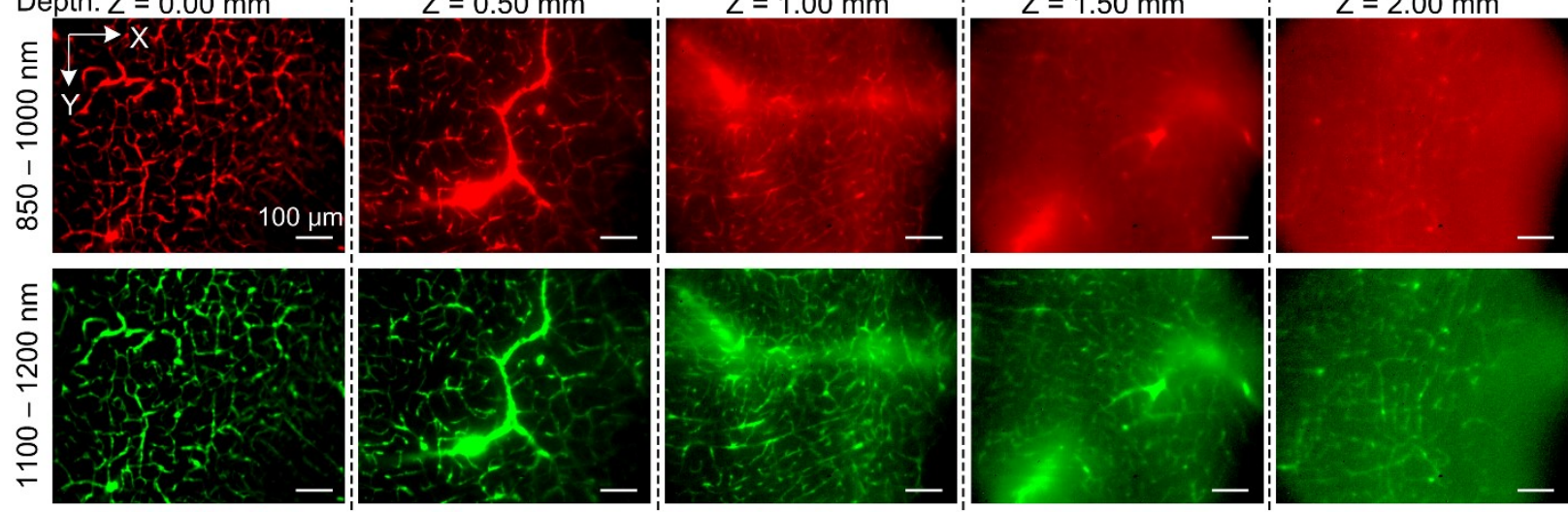

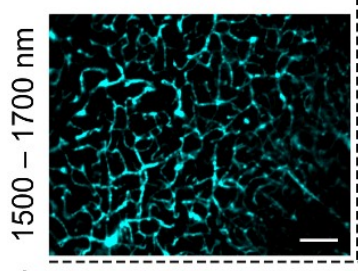

$\frac{d}{\dot{j}} 250$

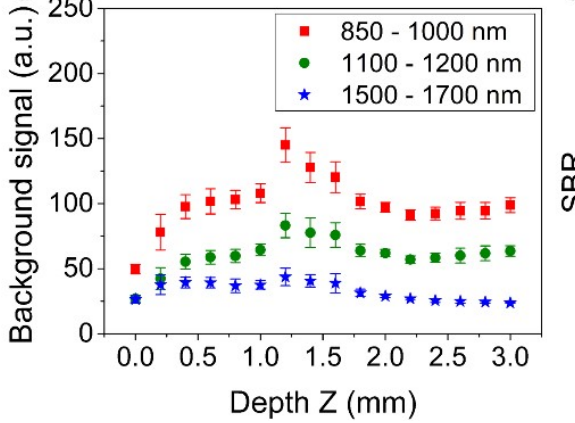

Depth Z (mm)

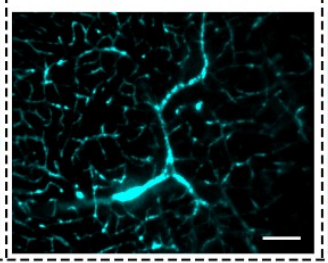

e
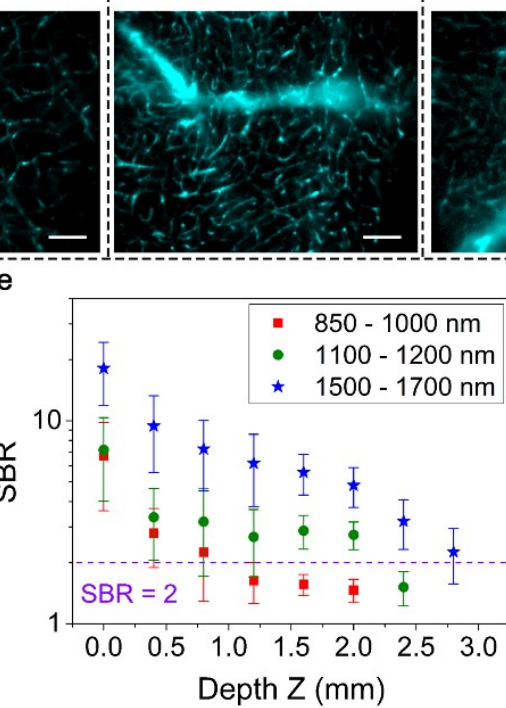
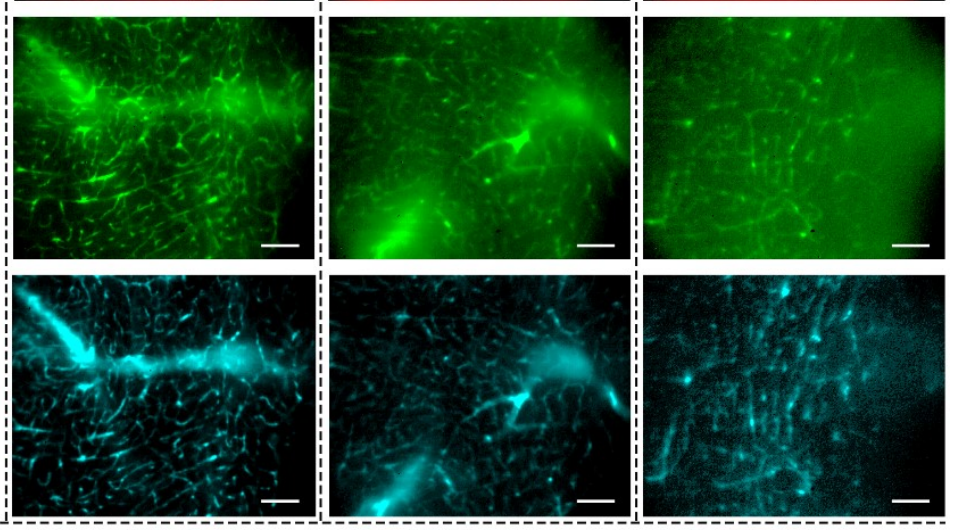

f

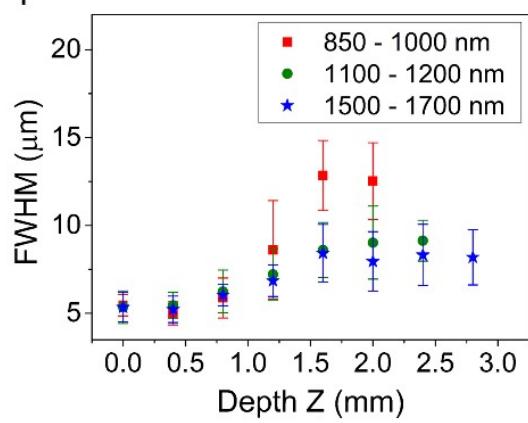

Figure 1 | Light sheet microscopy in various NIR 850-1700 $\mathbf{~ m m}$ emission sub-regions. (a) A simplified schematic of the NIR LSM. (b) Fluorescence emission spectra of p-FE and PEGylated $\mathrm{PbS} / \mathrm{CdS}$ core/shell quantum dots (see Supplementary Fig. 2 for excitation spectra). (c) Light-sheet optical sectioning mouse brain vasculatures at various depths in NIR-I, NIR-IIa and NIR-IIb emission regions using the same $785 \mathrm{~nm}$ light sheet illumination (also see Supplementary Video 1). The mouse brain tissue sample was prepared by intravenous injection of p-FE (emission: 850$1000 \mathrm{~nm}$ and 1100-1200 nm) and PEGylated PbS/CdS CSQD (emission: 1500-1700 nm) at 5-min 
interval. The mouse was scarified $30 \mathrm{~min}$ post injection of the probes while still in circulation. The mouse brain was taken out, fixed and preserved in glycerol for ex vivo imaging. See Supplementary Figs 4 and 5 for imaging details. The color bar range for each image is shown in Supplementary Fig. 3. Comparison of (d) background signal, (e) signal-to-background ratio and (f) FWHM of smallest vessels at various depths. Background was measured from randomly selected area without vasculatures. SBR is the ratio of fluorescence signals in randomly selected vasculatures over the background. (d-f) Error bars representing standard deviation were derived from analyzing $~ 10$ target data at every depth. A $10 \mathrm{X}(\mathrm{NA}=0.25)$ imaging objective and a $5 \mathrm{X}$ illumination objective (effective NA $=0.039$, light sheet waist $w=\sim 15.2 \mu \mathrm{m}$ and Rayleigh length $b=\sim 1258.2 \mu \mathrm{m}$ for 785-nm excitation, see Methods for light sheet shape analysis) were used in these experiments. Scale bars are $100 \mu \mathrm{m}$ for all images in (c). 


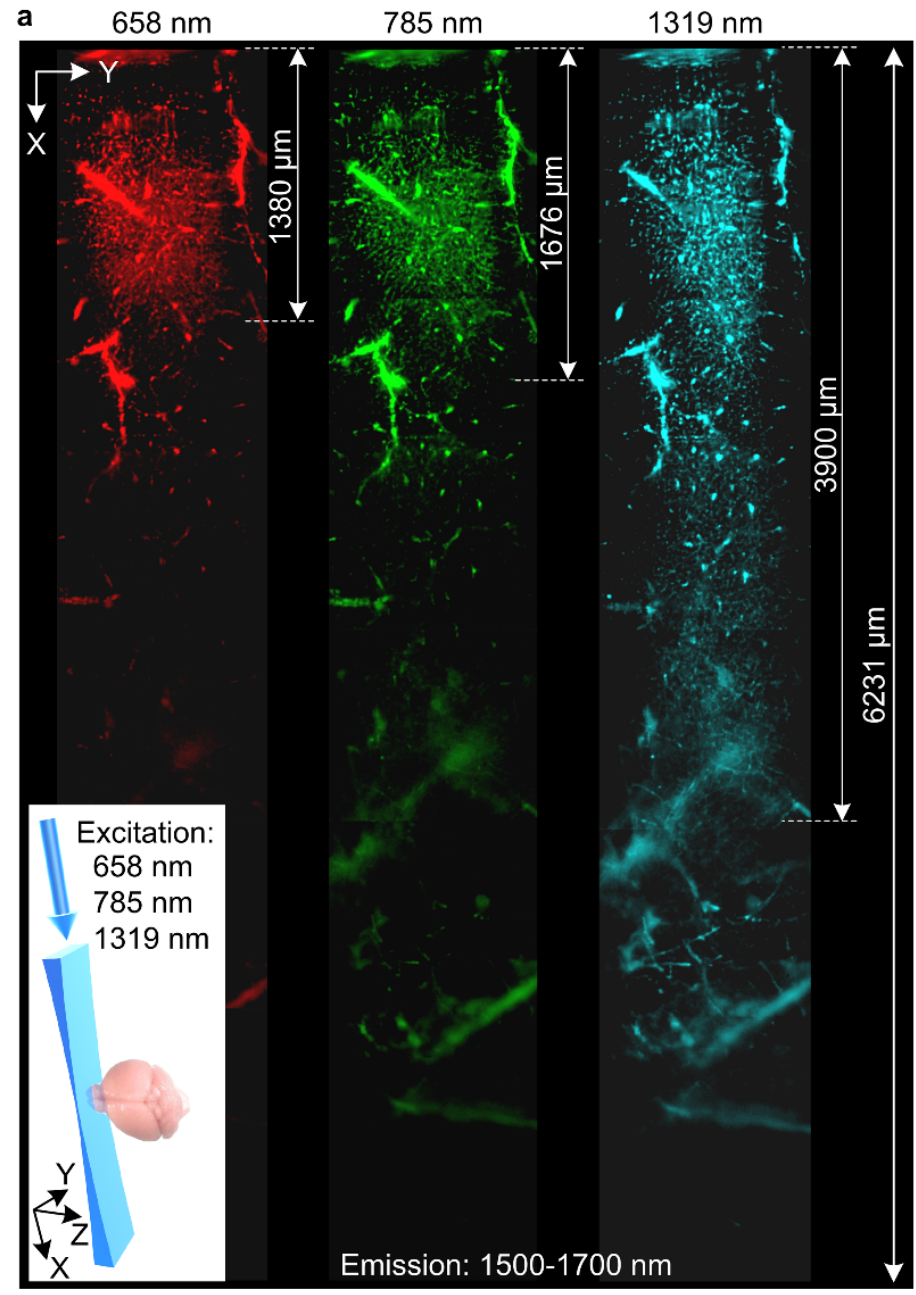

b

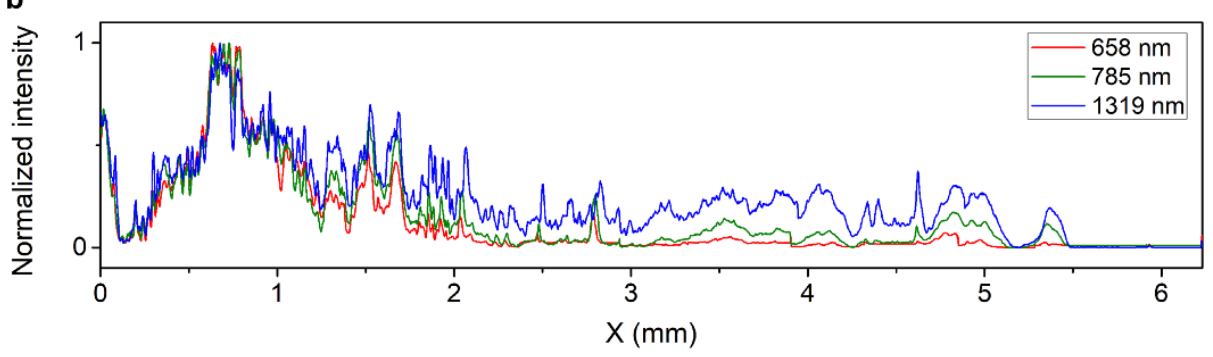

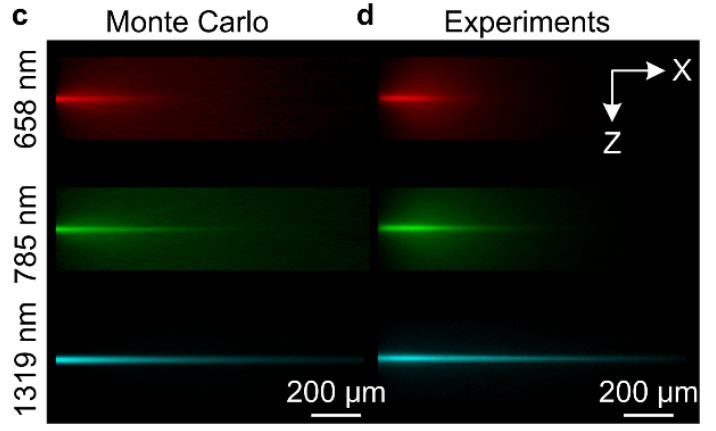
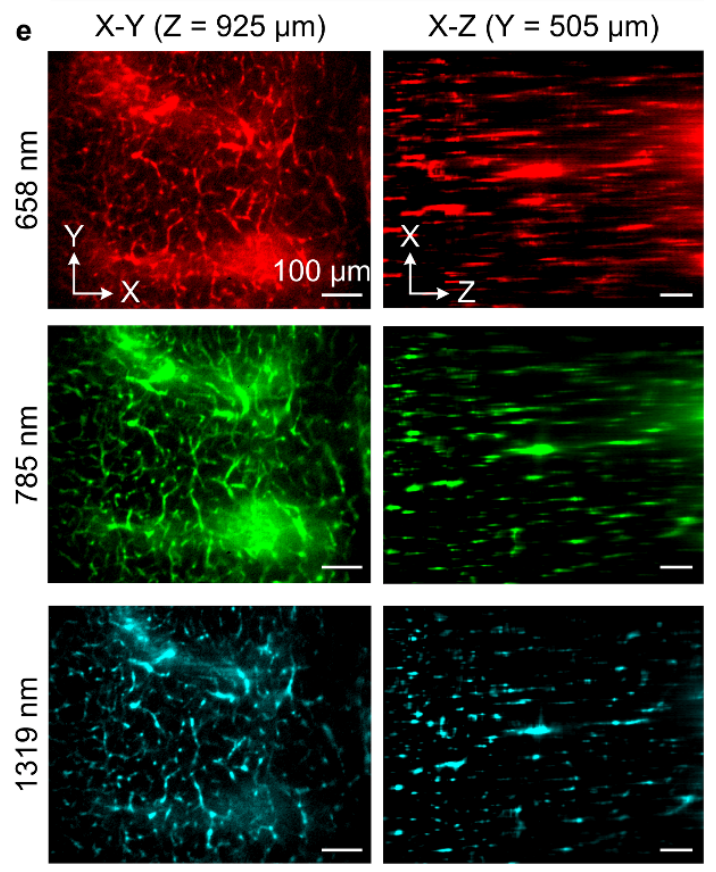

f

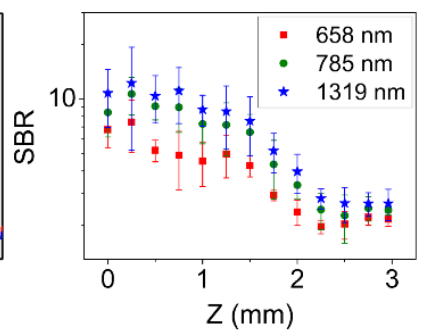

Figure 2 | Propagation of light sheet excitation with progressively longer wavelength up to $1319 \mathrm{~nm}$ in brain tissues. (a) $X-Y$ images of $1500-1700 \mathrm{~nm}$ quantum dot fluorescence in the vasculatures of a fixed brain tissue at a depth $Z=\sim 200 \mu \mathrm{m}$ under $658 \mathrm{~nm}, 785 \mathrm{~nm}$ and $1319 \mathrm{~nm}$ light sheet illumination as shown in the inset. The brain was taken out from a mouse 30 min after intravenous injection of PEGylated $\mathrm{PbS} / \mathrm{CdS} \mathrm{CSQD}$, fixed and preserved in glycerol before imaging. 6 images were taken along $X$ and stitched together for each light sheet. (b) Normalized sum intensity along $Y$ direction of images in (a) as a function of propagation distance $(X)$. (c) Monte Carlo simulations and (d) experimental results showing the $X$-Z propagations of different wavelengths light sheets in $2.5 \%$ intralipid tissue phantom (mimicking the brain) containing 
PEGylated PbS/CdS CSQD (emission: $1500-1700 \mathrm{~nm}$ ). Scattering coefficient $\mu_{\mathrm{s}}=109.3 \mathrm{~cm}^{-1}, 73.5$ $\mathrm{cm}^{-1}$ and $20.5 \mathrm{~cm}^{-1}$ and anisotropy $g=0.72,0.64$ and 0.34 were used to simulate $658 \mathrm{~nm}, 785 \mathrm{~nm}$ and $1319 \mathrm{~nm}$ excitation conditions respectively (see Supplementary Table 1). (e) Left: $X$ - $Y$ images of quantum dot 1500-1700 nm fluorescence in brain vasculatures taken at $Z=925 \mathrm{~m}$ under excitations by $658 \mathrm{~nm}, 785 \mathrm{~nm}$ and $1319 \mathrm{~nm}$ light sheets respectively (also see Supplementary Video 2). Right: images along the $X-Z$ plane at a fixed $Y$, reconstructed from $X-Y$ images at various depth $Z$ (also see Supplementary Video 3). A 10X, 0.25-NA detection objective was used and LS excitation was generated by a $5 \mathrm{X}$ illumination objective with an effective NA of $\sim 0.039$ (see Methods for experimental details and Supplementary Fig. 7 for light sheet shape analysis). (f) Comparison of SBR for $X$ - $Y$ images recorded at different depth for $658 \mathrm{~nm}, 785 \mathrm{~nm}$ and $1319 \mathrm{~nm}$ excitation. About 10 randomly selected vasculatures and 10 areas without vasculatures were analyzed to calculate SBR at each depth. Error bars represent standard deviation. Scale bars, 200 $\mu \mathrm{m}(\mathbf{c}, \mathbf{d})$ and $100 \mu \mathrm{m}(\mathbf{e})$. 
a

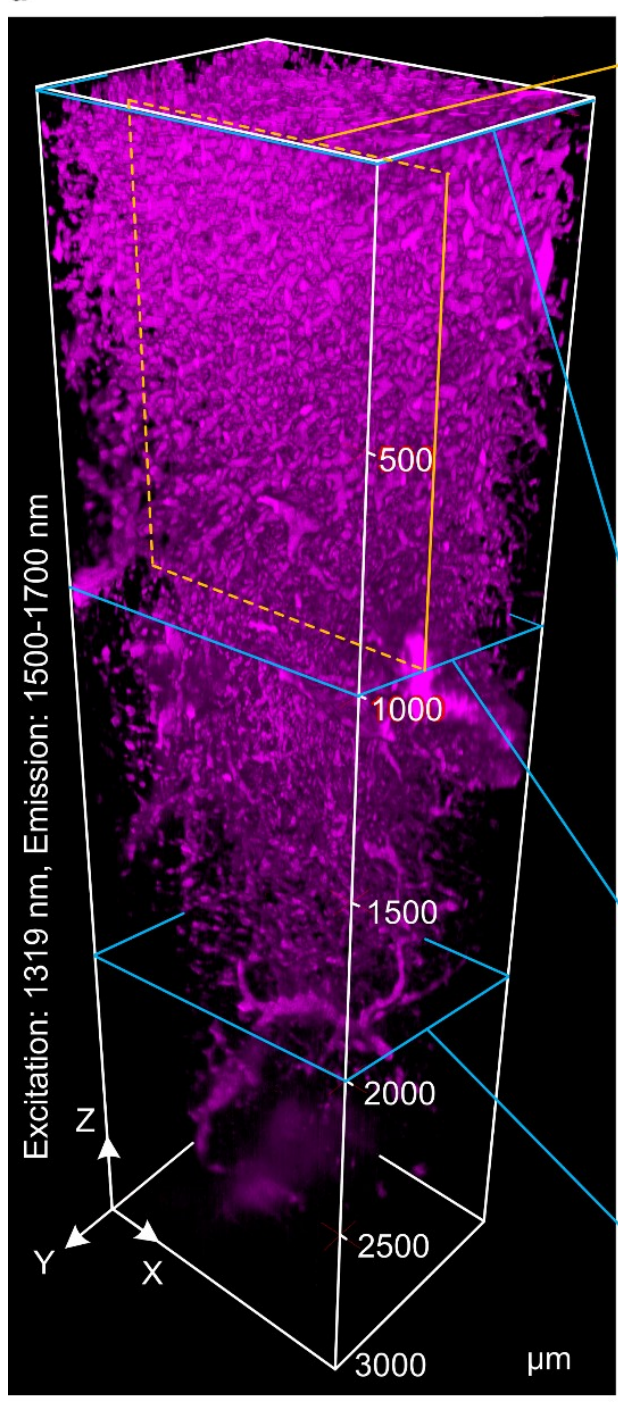

b

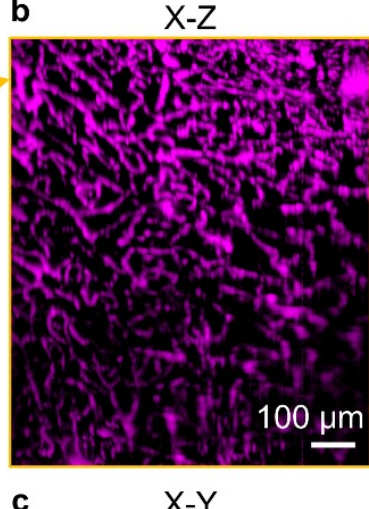

C
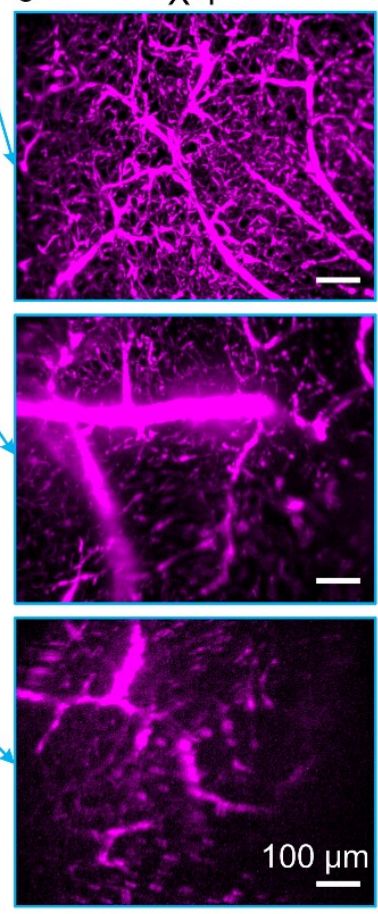

d

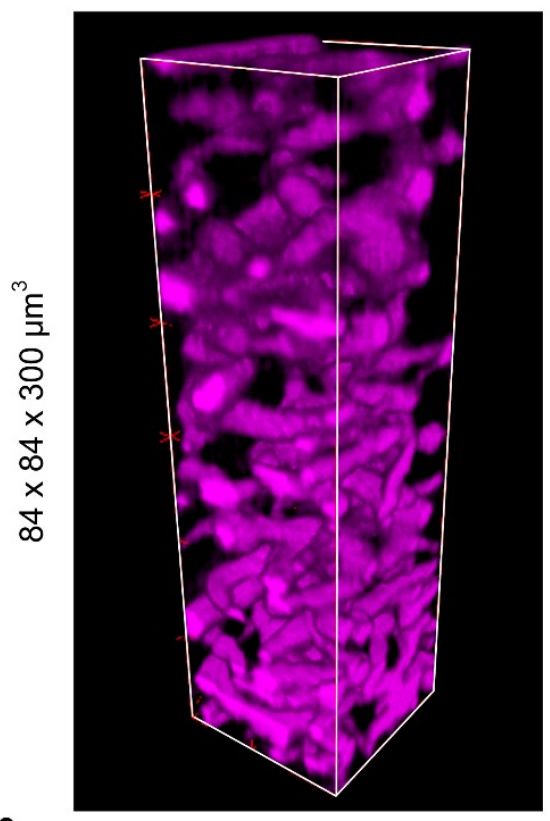

e

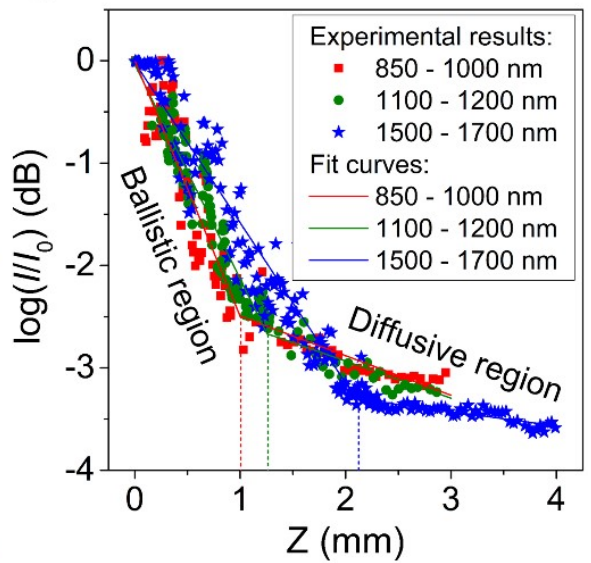

Figure 3 | Volumetric 1500-1700 nm fluorescence imaging of mouse brain sectioned by a 1319 nm light sheet. (a) 3D rendering of PEGylated PbS/CdS CSQD labelled vasculatures in mouse brain. 1500-1700 nm fluorescence was collected at $1319 \mathrm{~nm}$ excitation by a 10x detection objective. A $5 x$ illumination objective (with an effective NA $=\sim 0.051$ ) was used to generate LS excitation (also see Supplementary Video 4). The scanning increment along $Z$ was $3 \mu \mathrm{m}$. The excitation power $(\sim 8 \mathrm{~mW})$ and the exposure time $(0.8 \mathrm{~s})$ were kept constant during entire sectioning. (b) Maximum-intensity $Y$-projection (50 $\mu \mathrm{m}$ in thickness along $Y$, the maximum-intensity $Y$-projection took the brightest pixel in $X-Z$ layers through $50-\mu \mathrm{m} Y$ distance and displayed the maximum intensity values in the final 2D $X$ - $Z$ image) and (c) maximum-intensity $Z$-projections for a $150 \mu \mathrm{m}$ thick volume along $Z$ at $Z=0 \mu \mathrm{m}, 1000 \mu \mathrm{m}$ and $2000 \mu \mathrm{m}$, respectively. (d) 3D rendering of a smaller region in (a). (e) Maximum fluorescence intensity $(I)$ in different emission regions detected at various depths $(Z)$ in the mouse brain. $I_{0}$ is the fluorescence intensity at $Z=0 \mu \mathrm{m}$. In these experiments, the brain was taken out from a mouse intravenously injected with p-FE (excitation: $785 \mathrm{~nm}$, emission: 850-1000 nm and 1100-1200 nm) and PEGylated PbS/CdS CSQD (excitation: 
bioRxiv preprint doi: https://doi.org/10.1101/447433; this version posted October 18,2018 . The copyright holder for this preprint (which was not certified by peer review) is the author/funder. All rights reserved. No reuse allowed without permission.

785, emission: $1500-1700 \mathrm{~nm}$ ) with 5-min interval at 30 min post injection. Then the mouse brain was fixed and preserved in glycerol for ex vivo imaging. Scale bars, $100 \mu \mathrm{m}(\mathbf{b}, \mathbf{c})$. 

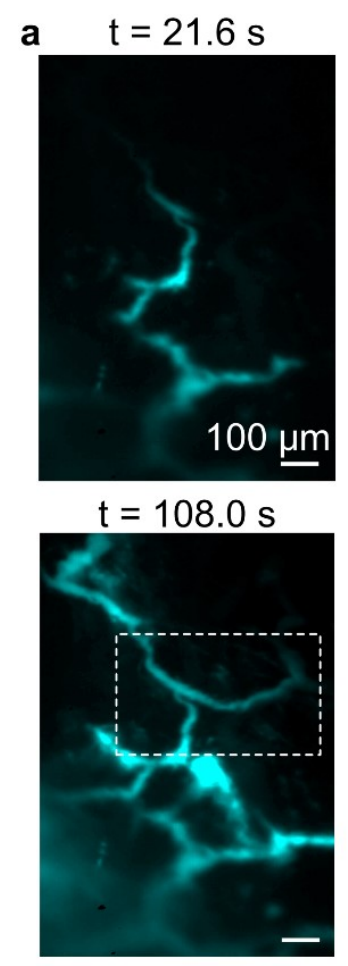

0

d

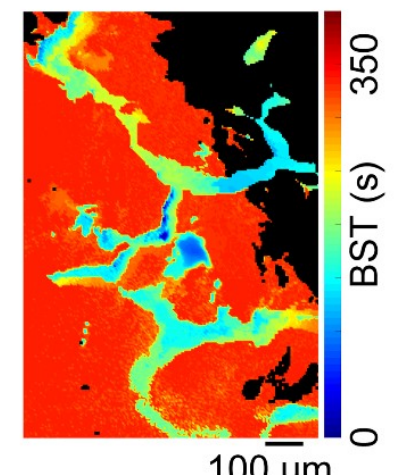

$100 \mu \mathrm{m}$

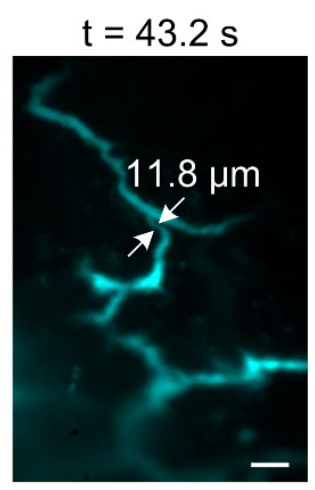

$t=216.0 s$

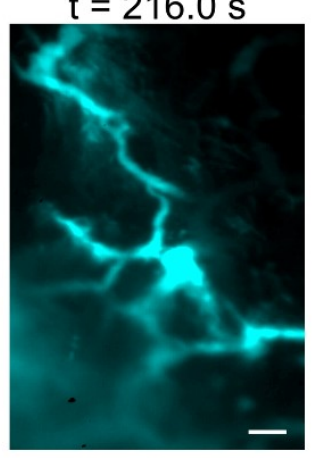

e

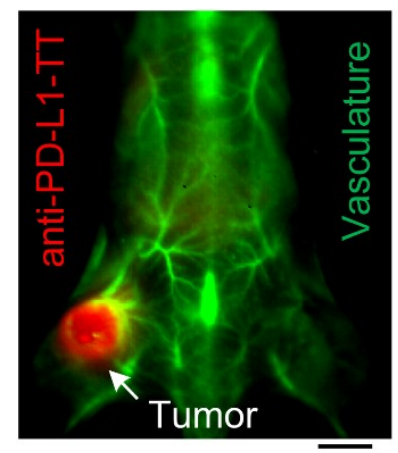

$5 \overline{\mathrm{mm}}$

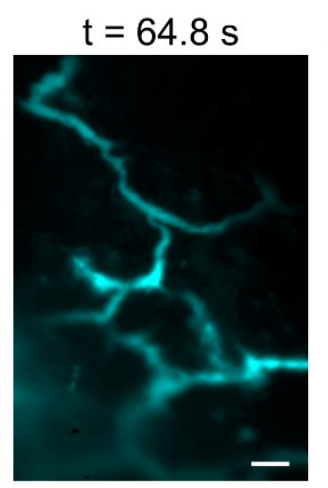

$t=324.0 s$

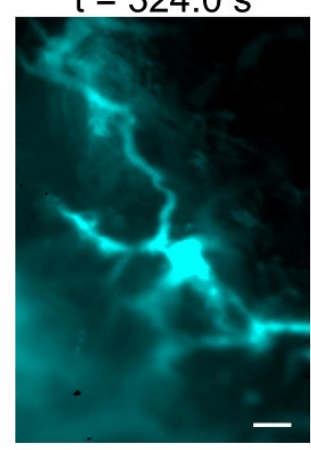

255
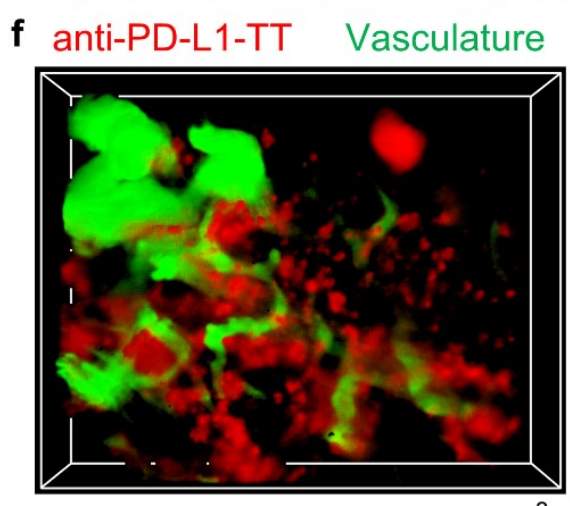

$810 \times 648 \times 300 \mu^{3}$

Figure 4 | In vivo NIR-II light sheet microscopy of xenograft tumors in mice. (a) Time-course (Supplementary Video 5) LSM of tumor vasculatures at a fixed illumination plane below the top of a xenograft MC38 tumor at $Z \sim 300 \mu \mathrm{m}$ after intravenous injection of p-FE (excitation: $785 \mathrm{~nm}$, emission: 1000-1200 nm). A 4X detection objective and a $5 \mathrm{X}$ illumination objective were used. (b) Schematic illustration of in vivo NIR LSM. (c) Abnormal blood flows in tumor vessels, showing on-off intermittency and direction reversal in the rectangular highlighted region in (A) and gradual extravasation into tumor space (Supplementary Video 5). Black arrows and red arrows represent blood with or without p-FE respectively. (d) A BST map (blood supply time, defined as the time at which the pixel intensity reached its maximum value relative to the fluorescence flow is first detected) showing highly heterogeneous blood perfusion in tumor vessels and slow, inhomogeneous extravasation behavior into tumor space. (e) Wide-field imaging of a mouse. The anti-PD-L1-TT probes (red color, excitation: $808 \mathrm{~nm}$, emission: 1000-1200 nm, exposure time 5 
$\mathrm{ms}$ ) were injected intravenously and kept circulation for $24 \mathrm{~h} .30 \mathrm{~min}$ prior to tumor imaging PEGylated PbS/CdS CSQD (green color, excitation: $808 \mathrm{~nm}$, emission: 1500-1700 nm, exposure: $5 \mathrm{~ms}$ ) was injected and imaged for overlaying with the tumor specifically labeled by the anti-PDL1-TT dye. (f) In vivo two-color 3D light sheet microscopy of anti-PD-L1-TT (red color, excitation: $785 \mathrm{~nm}$, emission: 1000-1200 nm, exposure: $0.8 \mathrm{~s}$ ) and vasculatures (green color, PEGylated $\mathrm{PbS} / \mathrm{CdS}$ CSQD, excitation: $1319 \mathrm{~nm}$, emission: $1500-1700 \mathrm{~nm}$, exposure: $0.8 \mathrm{~s}$ ) in a MC38 tumor using a $10 \mathrm{X}$ detection objective and a $5 \mathrm{X}$ illumination objective (Supplementary Video 6 ). The $Z$ scanning increment was $3 \mu \mathrm{m}$. The discrete red spots were down to $6 \times 6 \times 15 \mu \mathrm{m}^{3}$ in size, corresponding to PD-L1 expressing cells inside the tumor. No such spots were observed in tumor injected with TT dye without any anti-PD-L1 conjugated (Supplementary Fig. 13a). Scale bars, $100 \mu \mathrm{m}(\mathbf{a}, \mathbf{c}, \mathbf{d}) ; 5 \mathrm{~mm}(\mathbf{e})$. 